\title{
Mesenchymal Stem Cells as Targeted Cell Vehicles to Deliver Drug-loaded Nanoparticles for Cancer Therapy
}

Kytai Truong Nguyen*

Department of Bioengineering, UT at Arlington Joint Biomedical Engineering Program, UT Southwestern Medical Center at Dallas, 500 UTA BIvd, ERB-241, Arlington, TX 76019, USA

Mesenchymal Stem Cells (MSCs) have been gaining importance recently due to their multiple benefits in cancer therapy in addition to their advantages in other research fields such as tissue engineering and regenerative medicine. Results from several in vitro and in vivo studies have demonstrated that mesenchymal stem cells (MSCs) have the ability to home to tumor tissues. The tumor-tropic and migratory properties have made MSCs attractive to be engineered to express anti-proliferative, pro-apoptotic, and anti-angiogenic reagents for the treatment of localized and metastatic tumors [1-6]. Indeed, over the past few years, MSCs have been investigated and successfully used as gene therapy vehicles for cancer treatments [5]. This success suggests that MSCs could also serve as targeted carriers to deliver therapeutic agents including chemotherapy compounds to treat cancers. Since low targeting efficiencies and short half-lives of several cancer drugs are the major limitations for cancer therapy, using MSCs to carry and deliver drug loaded nanoparticles to cancer cells specifically might overcome these limitations.

Compared to other vehicles and/or delivery platforms as therapeutic carriers, MSCs might have more advantages. First, tumortropic properties would make MSCs accumulate specifically at the tumor site for better delivery of therapeutic reagents if using them as carriers. Beside tumor homing, one major advantage of MSCs is their ability to modulate the responses of the immune system or to have hypo immunogenic properties. These properties will help in increasing MSC survival after transplantation. Another major advantage of using these cells as vehicles to carry therapeutic reagents is that the potential use of autologous stem cells faces no issue with the immune responses and would increase cell survival after transplantation, leading to more effective delivery. It is also easy to obtain MSCs as they are present in many sources such as brain, liver, kidneys, pancreas, lungs, bone marrow, muscle, thymus, skin, adipose tissues, and umbilical cords. These advantages suggest that MSCs can be efficiently used as vehicles to carry and deliver therapeutic reagents for cancer therapy. Recently, Gao et al. [2] has provided a very good summary on using MSCs as targeted delivery vehicles for anti-cancer drug loaded Nanoparticles (NPs). Challenges faced when using MSCs for this application and strategies to overcome them have also been discussed in this article. These include strategies to improve NP uptake by MSCs, to maintain MSC viability while carrying cytotoxic anti-cancer drugs, to control the release profiles of drug-loaded NPs, and the fate of MSCs in vivo.

Although the use of MSCs is a promising means for targeted drug delivery vehicles for cancer therapy, this strategy might have several unknown answers to the use of MSCs as drug-loaded NP vehicles in humans. First of all, can all MSCs loaded with drug-encapsulated nanoparticles be specifically targeted to certain cancers for drug delivery without affecting healthy tissues? What happens if some cells are not homing to the tumor site, but are homing to the healthy tissues? What damages could these cells do to the healthy organs? There is also a conflicting result on the involvement of MSCs on enhancing tumor growth and malignancy. Some studies have shown that MSCs induce tumor growth most likely through their immune modulatory and pro- angiogenic properties, while other studies have demonstrated that MSCs either have no effect on tumor growth or demonstrate inhibition of tumor growth $[2,5]$. Thus there is still an uncertainty on whether MSCs could turn around to facilitate tumor growth and development. How dangerous would it be if these cell vehicles underwent spontaneous cell transformation involving tumor growth?

There are also several problems associated with such a new therapy that need to be solved prior to its use. One of them is to determine the effective delivery protocol including factors such as route of administration and cell concentration to have the optimal therapeutic effect. Systemic injection of cells (even though MSCs) through the blood stream might have some cells end up in the lung and spleen, requiring an increase in the number of cells administered for an effective therapy. This increase might not be feasible and practical Although MSCs can be easily extracted from different sources as stated earlier, they are found in small quantities in these sources whereas their growth and division efficiencies are significantly reduced once they are extracted from these sources. The use of these MSCs as a vehicle for drug-loaded NPs might require large-scale expansion of MSCs to produce clinical effectiveness, yet the laboratory culture of these cells in a large quantity might be a very challenging task. Further studies using large animal models are required to determine the long-term safety and efficacy of ex vivo expanded MSCs for use as a drug-loaded NP vehicle. It also remains to be determined the absolute number of MSCs and the involved molecular mechanisms and/or factors that promote MSC mobilization and homing to tumors to have the therapeutic effect for cancer treatments. Besides the tumor-tropic properties, MSCs might also contribute to tumor angiogenesis and be responsible for the growth of various tumors. These properties might dampen the enthusiasm for using these cells as drug and/or gene therapy delivery vehicles. Several strategies that allow us to impair the pro-neoplastic properties of MSCs while taking their tumor-tropic advantages for targeted drug/gene delivery to tumors need to be investigated. One of such strategies has been proposed, and that is to selectively eradicate MSCs after the delivery process by turning on the suicide gene in MSCs after they have done their job [2].

In all, the use of MSCs as drug-loaded NP vehicles will require a

${ }^{*}$ Corresponding author: Kytai Truong Nguyen, Department of Bioengineering UT at Arlington Joint Biomedical Engineering Program, UT Southwestern Medica Center at Dallas, 500 UTA Blvd, ERB-241, Arlington, TX 76019, USA, Tel: +1-817272-2540; Fax: +1-817-272-225; E-mail: knguyen@uta.edu

Received December 21, 2012; Accepted December 23, 2012; Published December 26, 2012

Citation: Nguyen KT (2013) Mesenchymal Stem Cells as Targeted Cell Vehicles to Deliver Drug-loaded Nanoparticles for Cancer Therapy. J Nanomed Nanotechol 4:e128. doi:10.4172/2157-7439.1000e128

Copyright: (c) 2013 Nguyen KT. This is an open-access article distributed unde the terms of the Creative Commons Attribution License, which permits unrestricted use, distribution, and reproduction in any medium, provided the original author and source are credited. 
Citation: Nguyen KT (2013) Mesenchymal Stem Cells as Targeted Cell Vehicles to Deliver Drug-loaded Nanoparticles for Cancer Therapy. J Nanomed Nanotechol 4:e128. doi:10.4172/2157-7439.1000e128

Page 2 of 2

compromise between its benefits, stem cell number requirements, costs, and safety issues to make it sufficiently attractive to pharmaceutical companies for investigating its development. There is no doubt that MSCs have the capacity for tissue regeneration and have a major impact in the tissue-engineering field; however, we are still a long way from being able to use these cells as targeted vehicles to deliver drugloaded NPs for cancer therapy, although this strategy is novel.

\section{References}

1. Galderisi U, Giordano A, Paggi MG (2010) The bad and the good of mesenchymal stem cells in cancer: Boosters of tumor growth and vehicles for targeted delivery of anticancer agents. World J Stem Cells 2: 5-12.
2. Gao Z, Zhang L, Hu J, Sun Y (2012) Mesenchymal stem cells: a potentia targeted-delivery vehicle for anti-cancer drug, loaded nanoparticles. Nanomedicine

3. Hu CC, Ke YQ, Sun XL, Jiang XD, Xu RX, et al. (2009) Human mesenchymal stem cells-like cells as cellular vehicles for delivery of immunotoxin in vitro. Biotechnol Lett 31: 181-189.

4. Hu YL, Fu YH, Tabata Y, Gao JQ (2010) Mesenchymal stem cells: a promising targeted-delivery vehicle in cancer gene therapy. J Control Release 147: 154162.

5. Shah K (2012) Mesenchymal stem cells engineered for cancer therapy. Adv Drug Deliv Rev 64: 739-748.

6. Sun XY, Nong J, Qin K, Warnock GL, Dai LJ (2011) Mesenchymal stem cellmediated cancer therapy: A dual-targeted strategy of personalized medicine. World J Stem Cells 3: 96-103. 